dispersion, but was invisible in the large spectroscope. The wave length assigned to it by $m e$ is a mere eye estimate, and it could easily be the line 554 observed by Professor Vogel in several planetary nebulae. The line 5268 was visible in the large spectroscope with difficulty, and only one setting of the micrometer wire upon it was attempted.
But Professor Vogel has also observed a similar line at 527 in GC. 4373 and the Orion Nebula (Scheiner's Spectralanalyse, p. 248,250 ). I have not searched for lines at these places, since the wave lengths in the case of the two Nova lines are not well enough fixed to make a successful search of any value.

\begin{tabular}{|c|c|c|c|c|c|c|c|c|c|c|c|c|c|}
\hline $\begin{array}{l}\text { Orion } \\
\text { Nebula }\end{array}$ & $\left|\begin{array}{c}\text { GC. } 4390 \\
(\Sigma 6)\end{array}\right|$ & $\begin{array}{l}\text { NGC. } \\
7027 \\
\end{array}$ & $\begin{array}{r}\text { GC. } \\
4964 \\
\end{array}$ & $\begin{array}{r}\mathrm{GC} . \\
4373 \\
\end{array}$ & $\begin{array}{c}\text { Nova } \\
\text { Aurigae }\end{array}$ & Intensity & $\begin{array}{l}\text { Orion } \\
\text { Nebula }\end{array}$ & $\left|\begin{array}{c}\text { GC. } 4390 \\
(\Sigma\end{array}\right|$ & $\begin{array}{l}\text { NGC. } \\
7027\end{array}$ & $\begin{array}{l}\text { GC. } \\
4964\end{array}$ & $\begin{array}{r}\text { GC. } \\
4373\end{array}$ & $\begin{array}{c}\text { Nova } \\
\text { Aurigae }\end{array}$ & Intensity \\
\hline$D_{3}$ & 5876 & $D_{3}$ & - & - & - & - & - & - & - & - & - & $45 \mathrm{I}$ & 0.1 \\
\hline- & - & $575^{I}$ & - & - & $575^{\circ}$ & $\mathbf{I}$ & 4473 & 4473 & - & 4472 & 4472 & 4466 & 0.1 \\
\hline - & - & 5412 & - & 一. & - & - & $439^{\circ}$ & 4390 & - & - & - & 438 & 0.1 \\
\hline- & - & 5313 & 532 & - & - & - & $43^{6} 3$ & 4364 & $43^{6} 3$ & $43^{64}$ & $43^{6} 3$ & $435^{8}$ & 0.8 \\
\hline 5007 & 5007 & 5007 & 5007 & 5007 & 5002 & 10 & $434 \mathrm{I}$ & 4341 & $434 I$ & 434 I & $434 \mathrm{I}$ & 4336 & 0.1 \\
\hline $495^{8}$ & $495^{8}$ & $495^{8}$ & $495^{8}$ & $495^{8}$ & 4953 & 3 & 427 & - & - & - & - & 426 & 0.1 \\
\hline 4862 & 4862 & 4862 & 4862 & 4862 & 4857 & I & 424 & - & - & 一 & - & 423 & 0.1 \\
\hline- & 4743 & 4743 & 4744 & - & - & - & 4102 & 4102 & 4102 & 4102 & 4102 & 4098 & 0.2 \\
\hline $47 \times 3$ & 4714 & 4716 & $47 I 4$ & - & $47 \mathrm{I}$ & 0.1 & 4067 & - & - & 4067 & 4067 & - & - \\
\hline- & 4687 & 4688 & 4686 & - & $468 \mathrm{I}$ & 0.4 & 4026 & 4026 & - & 4026 & 4026 & - & - \\
\hline $466 \mathrm{r}$ & 4663 & 一 & 4663 & 一 & - & - & 3969 & $39^{69}$ & - & 3969 & 3969 & 396 & Trace \\
\hline- & 4637 & $4^{6} 3 I$ & 4640 & - & 4630 & 0.7 & 3889 & - & - & - & 3888 & - & - \\
\hline - & 46 I0 & - & - & - & 460 & 0.1 & $3^{868}$ & $3^{868}$ & - & 3868 & 3867 & - & - \\
\hline- & 4595 & - & - & - & - & - & $3^{8} 3^{6}$ & - & - & - & - & - & - \\
\hline- & 4574 & - & - & - & - & - & & & & & & & \\
\hline
\end{tabular}

I an not certain that any of these nebulae contain a line near the wave length $45 \mathrm{I}$; but the other sixteen Nova lines are matched perfectly in one or more of them, allowing for the fact that the Nova lines were shifted about five tenth-metres (in August and September I892) towards the violet. The Nova spectrum certainly differs no more from the nebular spectra than the nebular spectra differ from each other.

It can safely be assumed that the lines at 4857 , 4336,4098 and 396 are the well-known hydrogen lines $H \beta, H \gamma, H \delta$ and $H$. The strongest lines in the spectrum, at 5002 and 4953 , then represent perfectly the positions and intensities of the well-known. ${ }^{\text {st }}$ and $2^{\text {nd }}$ nebular lines. There is no other type of spectrum in which these lines are known to exist. All the other well-determined Nova lines correspond perfectly to well-determined nebular lines. The presence of the chromosphere line 4472 and the four hydrogen lines must be considered as strengthening my argument, since they exist in the nebulae and are important factors in the definition of a nebular spectrum.

Nearly all the lines in the February 1892 spectrum of Nova corresponded to the prominent lines in the solar chromosphere, and vice versa. Aside from the hydrogen lines that correspondence has now become a striking discordance.

In view of the broad and multiple character of the prominent lines in the February 1892 spectrum we would naturally expect the present lines to be broad and complex. These characteristics of the lines in the early spectrum did not prevent us from arguing that they were chromospheric; why should the same characteristics of the present lines prevent us from arguing that they are nebular? If the present lines are multiple the problem only becomes more interesting and more hopeful of solution.

If the spectrum is not conceded to be nebular, I must ask what else we should expect to find in that spectrum if it were nebular.

Mount Hamilton, 1893 Aug. 1.

\title{
W. W. Campbell.
}

\section{Berichtigungen und Bemerkungen zur Bonner Südlichen Durchmusterung.}

\section{Von Fr. Deichmüller.}

Bei einer Vergleichung der übergreifenden Zone $\left(-19^{\circ}\right.$ bis $-22^{\circ}$ ) der Gill'schen photographischen Durchmusterung des südlichen Himmels mit der Bonner Durchmusterung hat Herr Prof. Kapteyn die nachfolgenden Abweichungen gefunden, und Herr Prof. Küstner hat sie mir zur Aufklärung nach den hiesigen Originalbeobachtungen übergeben.

Dass die fraglichen Fälle fast ausnahmslos $\mathrm{zu}$ den schwächsten der in den beiden Aufnahmen vorkommenden
Sterne gehören, zeugt wieder von der grossen Zuverlässigkeit der SD.; und wenn die Untersuchung auch einige weitere so seltene Fehler, wie sie Schönfeld SD. S. [19] angeführt hat, ergab, so liegen andererseits einige interessante Fälle vor, in denen Sterne der SD. auf den photographischen Platten fehlen, aber nach den Originalbeobachtungen und anderen Quellen als wohlverbürgt erscheinen. 


$$
\text { BD. }-19 \div 270 \text {. }
$$

Kapteyn findet statt $\delta=38: 9$ aus 4 Beob. 35:2.

Die BD. Zonen geben

$$
\begin{array}{rr}
\text { Z. } 144 & -19^{\circ} 34^{\prime} 4 \\
415 & 39.2
\end{array}
$$

Eine Rev. Beob. (Anschluss an Nr. 2.69) ergab 38:8, wonach $Z$. $144-I^{\mathrm{p}}$ corrigirt wurde, wodurch diese ebenfalls auf $3^{8: 8} \mathrm{kam}$. Nach K. sind aber Z. 415 und die Rev. Beob. um $+I^{\mathrm{p}} \mathrm{zu}$ corrigiren, also wird $\delta=-19^{\circ} 34^{\circ} 5$.

$$
\text { BD. }-1.9 \% 6001 \text {. }
$$

Kapteyn findet statt $\alpha=32$ so aus 2 Beob. $35^{\text {s. }} \cdot 5$.

$$
\begin{aligned}
& 33^{\circ} \text { giebt } \alpha=29^{\mathrm{s}} \cdot 6 \\
& 334
\end{aligned}
$$

Beide sind richtig gerechnet. Die Uhrzeit in Z. $33{ }^{\circ}$ ist also falsch notirt; $\alpha$ unsicher.

$$
\text { BD. }-20: 477 \text {. }
$$

K. findet $\delta$ statt $54: 2$ aus 4 Beob. zu 58:8.

$$
\text { Z. } \begin{array}{r}
10 \\
427
\end{array} \quad \delta=58: 4
$$

Eine Rev. Beob. (Anschluss an Nr. 476 u. 480) giebt $54: 4$, wonach $Z$. Io $+I^{p}$ corrigirt ist. Nach $K$. ist aber Z. $4^{27}$ und die Rev. Beob. um $-1^{p}$ zu corrigiren, und damit wird $\delta=5^{8: 6}$.

K. hat hier nur einen Stern.

$$
\text { BD. }-20^{\circ} 3343-44 \text { : }
$$

$\begin{array}{rrrrrrr}\text { Die Beob. lauten: } & \text { Z. } 174 & 77 \text { April } 15 & 9: 3 & 10^{\mathrm{h}} 58^{\mathrm{m}} 3^{\mathrm{m}} \cdot 3 & -20^{\circ} 5 \mathrm{r}: 9 & \text { Mittlere Luft } \\ & & 9.3 & 40.3 & 5^{2.3} & \\ & 503 & 80 \text { März I5 } & 9.3 & 40.6 & 51.6 & \text { Luft meist schön klar }\end{array}$

Da hier bloss ein Stern vorkommt, ist die Rev. Beob. angestellt

$$
\begin{aligned}
& \text { 82. April } 8 \quad 9^{\mathrm{m}} \cdot 3 \quad 1^{\mathrm{h}} 5^{8^{\mathrm{m}}} 3^{8^{\mathrm{s}} \cdot 7} \quad-20^{\circ} 5^{1: 8} \\
& 9.3 \quad 40.5 \quad 52.5
\end{aligned}
$$

Alles deutlich und richtig. Beide Sterne sind gesichert; das Fehlen von Nr. 3343 in Z. $5 \circ 3$ und bei $\mathrm{K}$. macht ihn der Veränderlichkeit verdächtig.

K. findet statt $\alpha=39$ s.o aus 3 . Beob. 46 s.2.

$$
\text { BD. }-20: 3648 \text {. }
$$

Der Stern ist Z. I $758^{\text {s }}$, in Z. $37^{2} \mathrm{I}^{\mathrm{s}}$ nachbeobachtet, die AR. also ganz unsicher und nach $\mathrm{K}$. zu corrigiren.

K. findet statt $\delta=36: 6$ aus 2 Beob. 43:7.

$$
\text { BD. }-20: 5106 .
$$

Die Beob. des BD. Sterns lauten:

$$
\begin{array}{rrrrrr}
\text { Z. } 70 & 7^{6} \text { Juni } 25 & 9^{\mathrm{m}} \cdot 5 & 18^{\mathrm{h}} 13^{\mathrm{m}} 23^{5} \cdot 9: & -20^{\circ} \cdot 36^{\prime} 6 & 8^{\mathrm{s}} \text { nachbeobachtet. } \\
& & 9.5 & 28.9: & 43.6 & 6^{5} \text { nachbeobachtet. Sehr klar. } \\
\text { Z. } 55^{8} & 80 \text { Aug. I } 2 & 9.5 & 27.7 & 36.2
\end{array}
$$

Der BD. Stern ist also gesichert und der K. Stern in Z. 70 ebenfalls beobachtet.

$$
\text { BD. }-20^{\circ} 5.198 \text {. }
$$

K. findet statt der BD. Position: $\quad 9^{\mathrm{m}} \cdot 6 \quad 18^{\mathrm{h}} 28^{\mathrm{m}} 5^{\mathrm{s}} \cdot 0 \quad-19^{\circ} 59^{\prime} \cdot 7$.

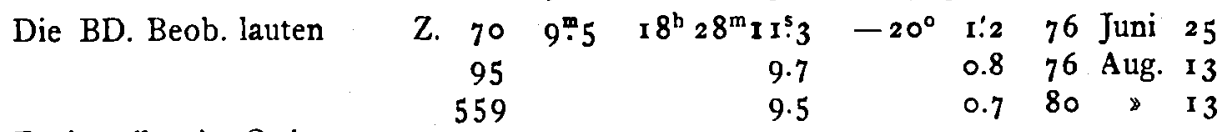

Es ist alles in Ordnung. 
K. findet auf den Platten nur einen Stern.

BD. -2 I $: 2599-600$.

$\begin{array}{rrrrrll}\text { Die Beob. lauten: } & \text { Z. } 293 & 9^{\mathrm{m}} \cdot 5 & 8^{\mathrm{b}} \mathbf{4 2}^{\mathrm{m}} 3^{8 \mathrm{~s}} \cdot 5 & -2 \mathrm{r}^{\circ} 5^{8: 0} & 7^{8} \text { April I } & \text { Klar } \\ & 9.5 & 40.5 & 56.7 & & \\ & 488 & 9.5 & 38.9 & 5^{8.7} & 80 \text { März } 6 & \text { Wechselnde Luft } \\ & 9.3 & 40.9 & 57.4 & & \\ & 12 & 10^{\mathrm{m}} & 39.9 & 57.8 & 76 \text { Jan. 27 } & \text { Klar } \\ & 10 & 40.4 & 57.4 & & \end{array}$

Die Existenz beider Sterne ist hiernach gesichert, und der eine für die photogr. Aufnahme zu schwach gewesen.*)

$$
\text { BD. }-21.2654 \text {. }
$$

K. findet statt $\delta=43: 5$ aus 3 Beob. $39: 7$.

Die Beob. lauten:

$\begin{array}{rlllrr}\text { Z. } 293 & 78 & \text { April I } & 10^{\mathrm{m}} & 8^{\mathrm{h}} 5 \mathrm{I}^{\mathrm{m}} 55^{\mathrm{s}} \cdot \mathrm{r} & -2 \mathrm{I}^{\circ} 43^{\prime} \cdot 4 \\ 488 & 84 & \text { März 6 } & 10 & 55 \cdot 3 & 43 \cdot 7\end{array}$

Es ist alles deutlich und richtig; $K$. und $B D$. werden zwei verschiedene Sterne haben.

$$
\text { BD. }-22: 2020-2 \mathrm{I} \text {. }
$$

K. findet hierfür :

$$
\begin{array}{rrr}
9^{\mathrm{m}} \cdot 2 & 7^{\mathrm{h}} 40^{\mathrm{m}} 29^{5} \cdot 9 & -22^{\circ} 3^{8 .} \mathrm{I} \\
8.9 & 33.4 & 3^{8.4}
\end{array}
$$

Die BD. Beob. lauten:

$\begin{array}{rcrr}\text { Z. } 6 & \text { 1 } 0^{\mathrm{m}} & 7^{\mathrm{h}} 40^{\mathrm{m}} 30^{\mathrm{s}} .2 & -22^{\circ} 42^{\prime} \cdot 2 \\ & 10 & 33 . \mathrm{x} & 43 . \mathrm{r} \\ \text { Rev, Beob. } & 9^{\mathrm{m}} \cdot 3 & 30.4 & 42.8 \\ 9.3 & 32.9 & 43.2\end{array}$

Die Theilstriche in Z. 6 lauten $-0 p_{7}-0$ p , eine Correctur von $+1^{p}$ ist also ausgeschlossen. Die Rev. Beob. (Anschlüsse an Nr. 2017, 18 u. 22) ist ebenfalls zweifelfrei. Die beiden Sterne sind also gesichert.

In Z. 288 findet sich aber eine Beob.

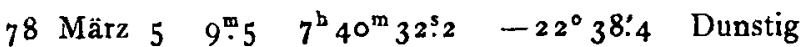

(Nr. 2020 u. 21 fehlen, 2017 u. 18 sind beobachtet). Dieser Stern ist offenbar identisch mit K.'s hellerem Stern, und sein Fehlen (in Z. 6 und) bei der Rev. Beob. macht ihn der Veränderlichkeit verdächtig.

$$
\text { BD. }-22: 2453-54 \text {. }
$$

K. hat hier nur einen Stern.

Die BD. Beob. lauten:

$$
\begin{array}{rrrr}
\text { Z. } 293 & 8^{\mathrm{h}} 5^{1^{\mathrm{m}} 48.3} & -22^{\circ} 57 ! 3 & \\
12 & 48.6 & 57.6 & \\
\text { Z. } 293 & 50.3 & 58.6 & \\
12 & 47 & 58.0 & \text { Diese Position }
\end{array}
$$

ist $10^{\text {s }}$ nachbeobachtet, trägt aber den Vermerk non idem $*$, so dass an der Existenz beider Sterne nicht zu zweifeln ist.

Bonn I 893 Juni 24.

$$
\text { BD. }-22: 3885 \text {. }
$$

K. findet statt $\alpha=14^{5} .1$ aus 3 Beob. 10.0 .

Die Rectascensionen lauten:

$$
\text { Z. } \begin{array}{rc}
60 & 6^{5} \\
317 & 11.3 \\
373 & 17.0
\end{array} \text { ist } 18^{s} \text { nachbeobachtet }
$$

und das Mittel aus den beiden letzten ist angesetzt. Die letzte Uhrzeit ist offenbar zu spät notirt, und die AR. unsicher.

$$
\text { BD. }-22^{\circ} \cdot 5342-43 \text {. }
$$

K. findet statt dieser beiden Sterne bei 4 Beob. nur den folgenden: $9{ }^{\mathrm{m}} \cdot 5 \quad 20^{\mathrm{h}} \circ^{\mathrm{m}} 16^{\mathrm{s}} 9 \quad-22^{\circ} 46^{\prime} \cdot 7$.

Die BD. Beob. lauten:

$\begin{array}{rcrc}\text { Z. } 101 & 9.5 & 20^{\mathrm{h}} 0^{\mathrm{m}} 9^{\mathrm{s}} \cdot 3 & -22^{\circ}[40: 6:] \\ 394 & 10^{\mathrm{m}} & 10.2 & 46.5 \\ \text { Z. } 101 & 9.5 & 16.2 & 42.8 \\ 394 & 9.5 & 14.7 & 43.8\end{array}$

und sind ferner durch die Beob. in Markree Cat. I und die Position auf Chacornac's Karte gesichert. Der photogr. Stern kommt in Markr. Cat. I ebenfalls vor:

$$
10^{\mathrm{m}} \quad 20^{\mathrm{h}} \mathrm{O}^{\mathrm{m}} 17^{\mathrm{s}} \quad-22^{\circ} 4^{6} \cdot 7
$$

und auf Chacornac's Karte (60)

$$
\text { 10.1 I } 1^{\mathrm{m}} \quad 20^{\mathrm{b}} \mathrm{O}^{\mathrm{m}} \mathrm{I} \eta^{\mathrm{s}} \quad-22^{\circ} 47^{\prime}
$$

Es fehlen auf den photographischen Platten also die beiden helleren Sterne.

$$
\text { BD. }-22: 5376 \text {. }
$$

K. findet statt dessen aus 5 Beob. :

$$
9.8 \quad 20^{\mathrm{h}} 6^{\mathrm{m}} 55^{\mathrm{s}} \cdot 5 \quad-20^{\circ} 38: 5
$$

Die BD. Beob. lauten :

Z. ro $\quad 9^{\mathrm{m}} .5 \quad 20^{\mathrm{h}} 6^{\mathrm{m}} 53^{\mathrm{s}} \quad-22^{\circ} 3^{8.2} \quad$ I $^{\mathrm{s}}$ nachbeobachtet

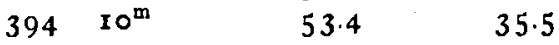

Da ferner Markr. I (S. 7 ) hat

$$
1 \mathrm{I}^{1} /^{\mathrm{m}} \quad 20^{\mathrm{h}} 6^{\mathrm{m}} 53^{\mathrm{s}} \quad-22^{\circ} 35^{\prime} \cdot 5
$$

und ebenso Chacornac an dieser Stelle einen Stern $12.13^{\mathrm{m}}$ hat, hat Schönfeld die Decl. in $Z$. I 1 I ausgeschlossen. Der $\mathrm{BD}$. Stern, der auf den photogr. Platten fehlt, ist also völlig gesichert, und der photogr. Stern kommt in den BD. Zonen auch einmal vor.

\section{Fr. Deichmüller.}

) Herr Prof. F. C. Kapteyn in Groningen bemerkt hierzu in einem Schreiben vom 2. Juli d. J.: ${ }$ Die Bemerkung, dass der eine Stern für die Aufnahme zu schwach gewesen sei, ist wahrscheinlich besser dahin abzuändern, dass er röthlich oder veränderlich sei. Die photographische Aufnahme dieser Gegend hat nämlich mehr als doppelt soviel Sterne als die SD., und der Stern, welchen die Platte hier aufweist, ist abber eine volle Grösse heller als die schwächsten der noch gemessenen Sterne. $K t z$. 